\title{
The Family Challenge of Caring for the Chronically Mentally Ill: A Phenomenological Study
}

\author{
Farshid Shamsaei, Fatemeh Cheraghi, ${ }^{2, *}$ and Ravanbakhsh Esmaeilli ${ }^{3}$ \\ ${ }^{1}$ Behavioral Disorders and Substance Abuse Research Center, Department of Nursing, Hamadan University of Medical Sciences, Hamadan, IR Iran \\ ${ }^{2}$ Chronic Diseases (Home Care) Research Center, Department of Nursing, Hamadan University of Medical Sciences, Hamadan, IR Iran \\ 3 Department of Nursing, Psychiatry and Behavioral Sciences Research Center, Addiction Institute, Mazandaran University of Medical Sciences, Sari, IR Iran \\ ${ }^{*}$ Corresponding author: Fatemeh Cheraghi, Chronic Diseases (Home Care) Research Center, Department of Nursing, Hamadan University of Medical Sciences, Hamadan, IR Iran. Tel: \\ +98-8138380150, Fax: +98-8138380447, E-mail: f_cheraghi@yahoo.com
}

Received: November 6, 2014; Revised: April 12, 2015; Accepted: August 22, 2015

Background: Family caregiving for patients with chronic mental illness is influenced by various factors such as political, socioeconomic, and cultural contexts as well as related policies and health services.

Objectives: The purpose of this study was to explore the challenges with which the family caregivers of patients with chronic mental illness have to contend.

Materials and Methods: The research design was qualitative with a phenomenological approach. The research population consisted of 16 long-term carers expressing interest in participating in the project. The carers were the family members of mentally ill relatives who collected their monthly medications at Farshchian Psychiatry Hospital in Hamadan in 2012. Purposive sampling was used to draw the sample. Data were collected by individual in-depth semi-structured interviews, which were tape-recorded and analyzed via Colaizzi's phenomenological method. Rigor was assessed regarding credibility, dependability, conformability, and transferability.

Results: Our findings highlighted 4 main themes, namely stress and emotional distress, need for education and information, socioeconomic effects and support, and physical strain.

Conclusions: Families experience frustrations when providing support and care to their mentally ill relatives. They, therefore, need appropriate support and intervention by mental health services.

Keywords: Caregivers; Family; Mental Disorders

\section{Background}

Mental illness is an important public health problem, both in its own right and because of its alliance to other chronic diseases with their concomitant morbidity and mortality. The world health organization (WHO) estimates that one of four families worldwide has at least one member suffering from a mental disorder and that mental disorders will constitute the largest global burden of disease by the year 2020. The onset and chronic presence of mental illness in the family can be a stressful event or a crisis for the family members (1).

Recent years have seen increasing awareness about the role of caregivers in the long-term management of psychiatric patients, and there is growing a body of literature on the caregiver burden, poor caregiver outcomes, lack of caregiver support, and equivocal success, with interventions aimed at alleviating the care-giving burden. It has also become clear that the emphasis in psychiatric rehabilitation needs to shift from a patient-focused approach to a combined patient- and caregiver-focused approach (2).

Families generally have little prior knowledge of mental illness and find that not only do they have to deal with the upheaval that often accompanies the disease but also many of the natural supports that they have come to rely on in difficult times are no longer available to them in their time of need. This is often due to a lack of awareness and often stigma about mental illness among typical sources of support such as extended family members, friends, and co-workers.

Carers play a vital role in supporting family members who are sick, infirm, or disabled. There is no doubt that the families of those with mental disorders are affected by the condition of their loved ones. Families not only provide practical help and personal care but also offer emotional support to their relative with a mental disorder. Therefore, the affected person is dependent on the carer, and their well-being is directly related to the nature and quality of the care provided by the carer (3).

These demands can create significant levels of stress for carers and can affect their overall quality of life, including work, socializing, and relationships. Research into the impact of caregiving shows that one-third to one-half of carers suffer significant psychological distress and experience higher rates of mental ill health than the general population. Being a carer can raise difficult personal issues about duty, responsibility, adequacy, and guilt. Caring for a relative with a mental health

Copyright (C) 2015, Mazandaran University of Medical Sciences. This is an open-access article distributed under the terms of the Creative Commons Attribution-NonCommercial 4.0 International License (http://creativecommons.org/licenses/by-nc/4.0/) which permits copy and redistribute the material just in noncommercial usages, provided the original work is properly cited. 
problem is not a static process since care recipients alter as their condition changes. The role of the carer can be more demanding and difficult if the care recipient's mental disorder is associated with behavioral problems or physical disability (4). Providing care for a patient with mental illness can be debilitating, stressful, and burdensome for the caregiver. In contrast, providing care to chronically ill or incapacitated family members may have a positive impact on family caregivers such as increased self-respect or self-satisfaction from fulfilling a responsibility (5).

Carers provide significant services at home to their relatives who need their help; however, lack of sufficient information and resources has always been an obstacle. As a result, carers are ill-prepared to play their roles as efficiently as possible (6). Iranian families are characterized by their intimate interpersonal relationships and many interactions among family members. Consequently, illness in one family member results in a substantial burden on the whole family. In addition, Iranian families report a low level of formal support services as compared with their Western peers (7).

Although the experience of caring for mentally ill relatives varies among families and cultures, a 1999 review article reported that family caregivers' largest challenges were providing assistance with daily activities (e.g., providing transportation, offering financial assistance, helping with housework, cleaning, and money management) and that stress associated with care included concerns about possible violence, embarrassing behaviors, and intra-family conflict (8).

A large majority of the studies about family caregivers' experiences have been carried out in Western cultures, with only a handful in developing nations. In fact, there has been no study to investigate the experiences of family caregivers in Iran. The findings of the research conducted in Western cultures may be difficult to extend to family caregivers in other cultures in attempting to understand their real caregiving experiences.

\section{Objectives}

The purpose of this study was to explore the challenges with which the family caregivers of individuals with chronic mental illness have to contend.

\section{Materials and Methods}

\subsection{Study Design}

Phenomenology served as the methodological framework for this study. The aim of phenomenological research is to examine the meaning of life through the interpretation of the individual's lived experience. A major concern of phenomenological research is to capture the totality of the human experience, with an emphasis on the meaning that social behavior has for the individual. Phenomenology is the study of lived experience in which the researcher is discovery-oriented (9) and is, as such, on a quest to seek answers to certain questions.

The participants were asked a common question: "What is your experience in caring for your mentally ill relatives?" The participants were encouraged to talk about their experiences, feelings about caring for their mentally ill relatives, and the support that they offered their mentally ill relatives to keep them in the community.

Lopez and Willis (2004) asserted that the phenomenological approach and nursing are a good fit because phenomenology seeks to understand the uniqueness of individuals and experiences (10). Investigators seeking to develop knowledge that embraces the ideals of holistic nursing encounter challenges in trying to understand human experiences in health and illness and identifying the caring needs of the nurses and clients who come together in temporary health care settings. The interactions inherent in holistic nurse-client transactions may be explored through phenomenological inquiry (11). Phenomenology provides the tools for deeply exploring human experience in order to understand the actual lived experience of a phenomenon by examining persons' accounts of their experiences.

\subsection{Participants and Setting}

Sixteen long-term carers expressed interest in participating in the project. Caregivers were identified as those family members who lived with the care recipient, provided ongoing support, and maintained regular contact. The carer support groups had members with varying years of caregiving experience. Purposive sampling was used to recruit informal caregivers identified as the family members of chronically mentally ill individuals residing in Farshchian Psychiatry Hospital in Hamadan, Iran. A psychiatric nurse at the facility initially contacted persons who met the following inclusion criteria: 1 ) family caregiver of a person with chronic mental illness including mood, anxiety, and schizophrenia disorders with the diagnosis of bipolar disorder as per the DSM-IVTR criteria, 2) male or female, 3 ) 18 years of age or over, and 4) Persian speaking. A research assistant subsequently contacted those individuals who verbally consented to be approached with more information about the study.

\subsection{Data Collection}

Data were collected through qualitative interviews and diaries. Following the consent process, the participants completed a demographic form. Face-to-face audio-taped interviews were held in a meeting room in Farshchian Psychiatry Hospital and the interviews lasted approximately 60-80 minutes.

The interview guide was composed of broad questions which provided the participants with the freedom to express their opinions and experiences. The initial 
questions began with queries about the participants' well-being such as "Tell me about your family member's illness". This approach helped the participants and the researcher to establish rapport with one another and facilitate communication. When the rapport developed between the interviewee and the interviewer, the questions proceeded to increasingly focus on the experience and challenges of caring for chronically mentally ill patients.

\subsection{Data Analysis}

Each transcribed interview was analyzed via Colaizzi's phenomenological method as described by Beck (12). The steps are as follows: 1) Read and re-read all the transcriptions to obtain a feeling for them. 2) Extract significant statements and phrases from the transcripts, pertinent to the research phenomenon. 3) Formulate meanings from the significant statements. 4) Organize the formulated meanings into a cluster of themes. 5) Integrate the results to date into an exhaustive description of the phenomenon. 6) Validate the exhaustive description by returning to the research participants. 7) If required, incorporate any new or relevant data obtained from participation validation. A summary of Colaizzi's method of analysis is depicted in Table 1 (13).

\subsection{Rigor}

To ensure rigor in the research findings, trustworthiness was established in accordance with the standard criteria for qualitative research, credibility, dependability, conformability, and transferability (13). To enhance credibility, we selected participants who were the family members of people diagnosed with chronic mental illness, in line with the accepted criteria. The interviews were conducted in a relaxed atmosphere facilitated by the first author's knowledge of the research area. The rich content of the interviews and the achieved saturation ensured that a satisfactory description was obtained of the caregivers' views about the condition of the person with chronic mental illness. The analysis was conducted by moving carefully back and forth between the data and the various steps in the analysis, and the analysis was discussed by the authors and agreement was reached at every step. Dependability was increased through discussion about similarities and differences in consistency and content over time and by having two main guiding questions to ensure that the same domains were dealt with in all the interviews. Numerous quotations from the participants were presented to shed light on their experiences of living with a family member with chronic mental illness. Clear descriptions of the selection, context, and the characteristics of the participants as well as the process of data collection and analysis facilitated the conformability and transferability of the findings.

\subsection{Ethical Considerations}

All the participants were informed about the purpose and the method of the study. They were informed that participation in the study was voluntary and that they could refuse to participate or withdraw from the study at any time without any personal consequences. The participants were reassured that their responses would be kept confidential and their identities would not be revealed in research reports or publications. Finally, the participants who agreed to take part in the study were asked to provide written consent.

\section{Results}

Sixteen caregivers were recruited. All the 16 participants were Iranian, and mothers accounted for the majority of the caregivers (37.5\%). The overall sample included 6 mothers, 2 siblings, 3 spouses (1 husband and 2 wives), 2 sons, and 3 daughters. More than two-thirds (69\%) of the participants were female. The majority of the participants (62.5\%) were married, while $12.5 \%$ were divorced, $18.7 \%$ were single, and 6.2\% were widowed. Most participants were employed and educated beyond high school. Caregiving experience ranged from less than 2 years to more than 14 years (Table 2 ).

Table 1. Summary of Colaizzi's Method of Analysis

\begin{tabular}{|c|c|}
\hline Steps in Analysis & Purpose \\
\hline 1. Reading and rereading descriptions & To acquire general feeling for experience \\
\hline 2. Extracting significant statements & $\begin{array}{l}\text { To generate information pertaining directly to the } \\
\text { phenomenon studied }\end{array}$ \\
\hline 3. Formulating meanings & $\begin{array}{l}\text { To illuminate meanings hidden in various contexts of the } \\
\text { phenomenon }\end{array}$ \\
\hline $\begin{array}{l}\text { 4. Categorizing into clusters of themes and validating with } \\
\text { the original text }\end{array}$ & To identify experiences common to all the participants \\
\hline 5. Describing & To generate a prototype of a theoretical model \\
\hline 6. Returning to the participants & To validate the findings \\
\hline $\begin{array}{l}\text { 7. Incorporating any changes based on the participants' } \\
\text { feedback }\end{array}$ & $\begin{array}{l}\text { To present a theoretical model that comprehensively reflects } \\
\text { the universal features of the phenomenon }\end{array}$ \\
\hline
\end{tabular}




\begin{tabular}{lc}
\hline Table 2. Demographic Characteristics of the Caregivers \\
\hline Variables & Values \\
\hline Age, $\mathbf{y}^{\mathrm{a}}$ & $47.1(6.7)$ \\
\hline Length of time, $\mathbf{y}^{\mathrm{a}}$ & $4.2(3.1)$ \\
\hline Gender $^{\mathrm{b}}$ & \\
\hline Female & $12(69.2)$ \\
\hline Male & $4(30.8)$ \\
\hline Marital status & \\
\hline Single & \\
\hline Married & $3(18.7)$ \\
\hline Divorced & $10(62.5)$ \\
\hline Widowed & $2(12.5)$ \\
\hline Employment & \\
\hline Retired & $1(6.2)$ \\
\hline Employed & $2(12.5)$ \\
\hline Unemployed & $3(18.7)$ \\
\hline Other & $3(18.7)$ \\
\hline Relationship to family member & \\
\hline Mother & $8(50)$ \\
\hline Wife & $2(12.5)$ \\
\hline Daughter & $3(18.7)$ \\
\hline
\end{tabular}

a Values are presented as mean (SD).

$\mathrm{b}$ Values are presented as No. (\%).
The following 4 themes were identified in the phenomenological analysis: stress and emotional distress, need for education and information, socioeconomic effects and support, and physical strain. Examples of theme clusters and the associated formulated meanings are demonstrated in Table 3.

\subsection{Stress and Emotional Distress}

Caring for a person with chronic mental illness is more stressful than caring for a person with a physical disability. All the participants identified that they experienced stress in their life due to their role as a caregiver for their family member with a mental illness. Individuals who experience the most caregiver stress are the most vulnerable to changes in their own health.

One participant spoke about experiencing stress due to his son's mental illness. He said, "I have felt excessive stress and sadness from all of this. It's just always on your mind-wondering what's going to happen next, what will happen when we're not around, or when we're not able to care for him".

While emotional distress issues were experienced by most of the participants, mental or emotional health issues were also common throughout the narratives. Many of the participants spoke of their personal battles against emotional distress and most were still taking psychiatric drugs. Speaking about emotional distress, one caregiver said, "Emotionally I'm dead. My life is in a shambles and I'm madly trying to fix it and I'm not getting anywhere, and I keep trying. All the time, I keep trying. I seem to be getting very, very depressed and upset. I don't really want to take more psychiatric drugs you know".

Some family caregivers felt worried and guilty, indicating a complex and somewhat conflicting emotional response.

"I felt sick and weak; I didn't want that to happen. As much as I knew that it was going to make him better, I didn't want him to be... I didn't want him in the hospital at all. I was relieved knowing that something was finally being done because like I said, it was a long process. It was a very long process".

Table 3. Examples of Theme Clusters and the Associated Formulated Meanings

\begin{tabular}{llc}
\hline Examples of Formulated Meanings & Theme Clusters & Emergent Theme \\
\hline Social isolation & & Socioeconomic effects and support \\
\hline Disliking social relationship & Social interaction & \\
\hline Disliking people' opinion of them & \\
\hline Tendency to talk less & \\
\hline Disliking compassionate people & Needs of supportive \\
\hline Disliking people questionings & \\
\hline Supportive health care providers & \\
\hline Supportive friends and relatives & \\
\hline Supportive in financial terms
\end{tabular}


Shamsaei F et al.

\subsection{Need for Education and Information}

Many of the family members interviewed believed that because they were the primary caregivers to their mentally ill loved ones, they needed information about the diagnosis, prognosis, and treatment. Furthermore, they expressed the desire to be included in treatment planning sessions so that they could provide consistent care that would parallel what mental health professionals sought to accomplish rather than find out what they had done or what they had not done was the complete opposite of what the mental health provider might have done.

"I grab pieces of information where I can get them. It would be nice to get reports from them [i.e., mental health professionals] to know that she [ie, daughter] is doing well and what the plan is for her. That would relieve a lot of our stress, but we don't get any kind of contact with any of them to know if things are moving forward or if she is doing better. For now I just use my instincts to know if things are getting better or not".

Information, support, and mutual trust are essential. Family members are often left very much in the dark. This puts more of a strain on them. Every decision should involve the family, especially if that is what the patients demand. Be that as it may, all too often that this is not the case.

One of the participants explained the situation as follows: "No education was given except for the interview that they normally conduct about when the illness started. I sometimes read books about the illness and learn how to care for these patients".

One daughter caregiver said, "I don't know what this illness really is. What worries me most is that I don't know if my behavior toward my brother is good." And a husband caregiver said, "The doctors explained to me something about what bipolar disorder is but very little on how to manage the patient at home.

"The family needs to be educated about the illness, and educated about what services are out there. Mental health professionals need to become partners with us in care. The help needs to be offered to families, not asking them to seek out all the help on their own".

\subsection{Socioeconomic Effects and Support}

The participants' experiences showed that the perceived need for social support among the family caregivers of people with a chronic mental illness is considerable. All the participants emphasized the benefits associated with having social support to assist them in their role and to provide empathy and encouragement as needed.

A mother providing care to her son struggling with a chronic mental illness agreed with the critical nature of having social support when providing care to a family member with a mental illness. She said, "Often times when a kid has a mental illness, you are so isolated. Just isolated. When my son was diagnosed with mental illness, it was stigmatized everywhere that we went from extended family to school, the doctor, even the psychiatrist. Social support was so important to me."

One participant said that friends and family members did not necessarily understand why the situation was stressful and how they could help. A father said, "I wouldn't say that I'm getting much support. My children visit occasionally, but I also understand that they have their own demanding lives and responsibilities. We can only have reasonable expectations."

Financial constraints were reported to be the major challenge to most of the participants in providing support to their mentally ill loved ones. Some depended on charity and loans from their relatives for survival as they had no source of income. Others were obliged to quit their job so that they could tend to their relatives.

The financial assistance that the families offered their mentally ill relatives in this study included giving them money to buy cigarettes and buying them food and clothes. One participant said, "Well, he is not using his borrowed money well. He is smoking too much. I tried to get him to quit smoking, but I failed. His money is spent on smoking. We are afraid to talk to him about the loan. He uses a card and is quite friendly outside, but at home he will not be all right if we tell him to buy food with the borrowed money."

Some family members also feel that logistics such as travel, meeting times, and money can affect the extent of the commitment a family member can make to care for a mentally ill loved one. The way in which each mental health professional accommodates these logistics will determine the role that family members can play in decision making and truly becoming an active, important member of the treatment team.

\subsection{Physical Strain}

Some participants did perceive that their immediate health problems were directly related to the stresses of caring for their mentally ill family member. Some participants believed that this stress manifested as conditions such as high blood pressure, migraine, chest pain, and gastritis and colitis. One participant said, "I couldn't sleep, I was worried. I had a terrible migraine and I was vomiting and all the rest. I've stomach pain; the anxiety symptoms have hit me."

\section{Discussion}

The findings of this study revealed various stress and emotional distress, need for education and information, socioeconomic effects and support, and physical strain that the family caregivers experienced in living with a chronically mentally ill family member.

Caregiving maintains all the features of a chronic stressor. It involves psychological and physical strain over long periods of time and has the capacity to spill over into various areas of life such as the workplace and family relations. In addition, it is frequently accompa- 
nied by high levels of unpredictability and often requires demanding vigilance. Caring for a family member with a mental health problem is a significant chronic stressor in humans. In fact, caregiver stress is currently seen as a human model of chronic stress in the stress literature (14).

Caregivers face many obstacles as they balance caregiving with other demands, including child rearing, career, and relationships. They are at increased risk for burden, stress, depression, and a variety of other health complications (15). The effects on caregivers are diverse and complex, and there are many other factors that may exacerbate or ameliorate how caregivers react and feel as a result of their role.

Some of the problems that a family carer has to contend with include not receiving proper education from health professionals about the illness, medication, and its side effects; attitudes of health professionals; and the patient's non-response to medication. In this study, most of the participants were not given any education about the mental illness and medication thereof.

Jubb and Shanley (2002) identified factors that militate against caregivers' contribution to the recovery of their family members, including failure of professionals to recognize the therapeutic benefits of caregivers and their involvement in the treatment programmed (16). Education is an important component of providing the families of individuals with a chronic mental illness with the tools to assist in the provision of care for their loved ones (17). Dreier and Lewis (1991) stated that when family members received information about their loved ones' diagnosis, as well as strategies to assist in managing the illness, feelings of mastery increased, while feelings of blame toward their family member and personal feelings of guilt decreased (18).

The family members interviewed for this study expressed their desire for mental health professionals to educate them about the etiology, treatment, and prognosis of the illness and be available to answer questions. Many family members do not know what the diagnosis of their mentally ill loved one is, nor do they know what medications their loved one is taking and what side effects these may have. They also do not know what changes they should be looking for when their loved one is put on certain medications.

The family members interviewed for this study reported that mental health professionals did not involve the family members in the treatment of their mentally ill relative or help address the needs of the caregiving family member. Furthermore, these participants never mentioned attending any educational programs developed by mental health professionals. All the participants agreed that these programs would be appreciated.

Caregivers need a great deal of support in different forms, be it practical or emotional support. Unfortunately, most of the caregivers in the present study reported that they did not receive sufficient support. Social support can be provided both informally (e.g., by family, friends, neighbors, and social groups) and formally (e.g., by professionals and agencies) (19).

There is some evidence in the literature that the amount and quality of social support available to caregivers is an important factor in moderating the impact of caregiving. However, other researchers have reported some inconsistencies in the mediating role of social support (20). Support can come in the form of instrumental support (helping with daily living needs and housework), emotional support, and informational support (information and knowledge from both health professionals and from those who have experienced similar situations). A positive relationship between social support and psychological well-being has generally been found. Support provides a buffer against burden and stress for caregivers by increasing the perception that resources are available to handle stress (19).

In the present study, the family caregivers of chronically mentally ill patients had financial problems. Caring for an individual with a chronic mental illness also requires notable financial commitment (20). Medical insurance may not cover certain types of treatment or medications.

The family may be put in the position of having to fund these components of treatment for their loved one with a chronic mental illness. Some chronic mental illnesses also manifest themselves by way of destructive spending habits or difficulty with budgeting/money management, providing another way that the family members of individuals with a chronic mental illness might experience financial hardship (21). Caring for an elderly family member demands a significant amount of energy from the caregiver, placing them at risk for physical and mental health decline as a result of the stress caused by caregiving (22).

All the participants in this study identified feeling physical strains due to their role as a family caregiver to their relative with a chronic mental illness. Physical strains are all consequences of the long-term experience of family caregiving.

The chronic stress of caregiving affects numerous dimensions of caregiving health, including self-reported health, health symptoms, illness, and medication use. A revealing study has shown that caregivers experience a one-third increase in negative health symptoms after assuming caregiving responsibilities. Unsurprisingly, caregivers use significantly more prescription medication than non-caregivers.

The detrimental physical effects of caregiving are generally less intensive than the psychological effects, regardless of whether they are assessed by global self-report instruments or physiological measures such as stress hormone levels. Although relatively few studies have focused on the association between caregiving and health habits, researchers have found evidence of impaired health behaviors such as neglecting their own health care appointments and eating a poor-quality diet among caregivers who provide assistance with basic activities of daily living like toileting and eating (2). One challenge 
that Iranian caregivers experienced was stigma. Stigma is a dynamic, multifaceted social process that has been consistently implicated as a key contributor to poor outcomes for many people who live with stigmatized health conditions such as mental illnesses (23).

Overall, the caregivers of relatives with mental disorders experience many psychological, physical, social, and economic problems and challenges. These include stress, emotional distress, lack of social support, disruption in social life, financial hardship, and need for information and education. Professional assistance, social and financial support by the government, and increasing caregivers' information and education are important in addressing these challenges.

Collaboration between family members and mental health professionals needs to consist of 3 key elements. Firstly, mental health professionals should recognize and respect the pivotal role that families play in the lives of their mentally ill loved ones and learn to value family members' judgments and ensure that their efforts are supported. Secondly, professionals should develop more opportunities, both formal and informal, to learn about the perspectives of families and to work together with family members as equals at various levels of care. And thirdly, mental health professionals also need to reinforce family strengths and make it possible to provide individualized care for each mentally ill adult and their family.

\subsection{Strengths and Limitations}

The choice to use a qualitative research modality to explore the research is a strong point. Relying on the participants' own words enabled the researcher to obtain more detailed and in-depth information about their experiences. Because the interviews were done in person, the researcher was also able to observe non-verbal behaviors to assist in eliciting appropriate clarifying questions or follow-up prompts. Nonetheless, the sample size and limited diversity in gender, age, race, and ethnicity affected the scope of the study findings. Future research using a larger and more diverse sample will enrich our understanding of the needs and contributions of family caregivers.

\section{Acknowledgements}

This study was supported by the research center for behavioral disorders and substance abuse in Hamadan university of medical sciences, Iran (Grant \# 903222865).

\section{Declaration of interest}

None declared.

\section{Authors' Contributions}

Farshid Shamsaei and Fatemeh Cheraghi designed the study, collected the data and drafted the manuscript. Ravanbakhsh Esmaeilli interpreted the clinical data, performed the data analysis and helped to draft the manuscript. All authors read and approved the final manuscript.

\section{References}

1. WHO. Promoting mental health: concepts, emerg $\neg$ ing evidence, practice. Switzerland: World Health Organization; 2004.

2. Burton LC, Zdaniuk B, Schulz R, Jackson S, Hirsch C. Transitions in spousal caregiving. Gerontologist. 2003;43(2):230-41.

3. Oyebode JR. Carers as partners in mental health services for older people. Adv Psychiatr Treatment. 2005;11(4):297-304.

4. Shah AJ, Wadoo O, Latoo J. Psychological distress in carers of people with mental disorders. Brit J Med Pract. 2010;3(3):ea327.

5. Kuuppelomaki M, Sasaki A, Yamada K, Asakawa N, Shimanouchi S. Family carers for older relatives: sources of satisfaction and related factors in Finland. Int J Nurs Stud. 2004;41(5):497-505.

6. Corsentino EA, Molinari V, Gum AM, Roscoe LA, Mills WL. Family caregivers' future planning for younger and older adults with serious mental illness (SMI). J Appl Gerontol. 2008.

7. Sharif F, Shaygan M, Mani A. Effect of a psycho-educational intervention for family members on caregiver burdens and psychiatric symptoms in patients with schizophrenia in Shiraz, Iran. BMC Psychiatry. 2012;12(1):48.

8. Baronet AM. Factors associated with caregiver burden in mental illness: a critical review of the research literature. Clin Psychol Rev. 1999;19(7):819-41.

9. Van Manen M. Researching Lived Experience: Human Science for an Action Sensitive Pedagogy. State University of New York Press; 1990.

10. Lopez KA, Willis DG. Descriptive versus interpretive phenomenology: their contributions to nursing knowledge. Qual Health Res. 2004;14(5):726-35.

11. Wojnar DM, Swanson KM. Phenomenology: an exploration. J Holist Nurs. 2007;25(3):172-80.

12. Colaizzi. Psychological research as the phenomenologist views it. In: Valle RS, King M, editors. Existential phenomenological alternatives for psychology. New York: Oxford University Press; 1978.

13. Polit D,F, Beck CT. In: Essentials of nursing research. 6, editor Philadelphia: Lippincott Williams \& Wilkins; 2006.

14. Wan N. The stress and stigma of caregiving. Mammoth Magazine. 2011;10:3.

15. Cassie K M, Sanders S. Familial caregivers of older adults. In: Cummings SM, Kropf, Nancy P, editors. Handbook of Psychosocia Interventions with Older Adults: Evidence-Based Approaches. Philadelphia: Haworth Press; 2008

16. Jubb M, Shanley E. Family involvement: the key to opening locked wards and closed minds. Int J Ment Health Nurs. 2002;11(1):47-53.

17. Tabatabainia MM. Listening to families' views regarding institutionalization \& deinstitutionalization. J Intellect Dev Disabil. 2003;28(3):241-59.

18. Dreier MP, Lewis MG. Support and psychoeducation for parents of hospitalized mentally ill children. Health Soc Work. 1991;16(1):11-8.

19. Shamsaei F, Mohamad Khan Kermanshahi S, Vanaki Z, Hajizadeh E, Hayatbakhsh MR. Experiences of family caregivers of patients with bipolar disorder. Asia Pac Psychiatr. 2010;2(4):201-7.

20. Savage $S$, Bailey $S$. The impact of caring on caregivers' mental health: a review of the literature. Aust Health Rev. 2004;27(1):111-7.

21. Lai. Thomson C. The Impact of Perceived Adequacy of Social Support on Caregiving Burden of Family Caregivers. Fam Soc J Contem Soc Serv. 2011;92(1):99-106.

22. Goodman H. Elderly parents of adults with severe mental illness: Group work interventions. J Gerontol Soc Work. 2005;44(12):173-88.

23. Shamsaei F, Mohamad Khan Kermanshahi S, Vanaki Z, Holtforth MG. Family Care giving in Bipolar disorder: Experiences of Stigma. Iran J Psychiatry. 2013;8(4):188-94. 